\title{
A one-level additive Schwarz preconditioner for a discontinuous Petrov-Galerkin method
}

Andrew T. Barker ${ }^{1}$, Susanne C. Brenner ${ }^{1}$, Eun-Hee Park ${ }^{2}$, and Li-Yeng Sung ${ }^{1}$

\section{A discontinuous Petrov-Galerkin method for a model Poisson problem}

Discontinuous Petrov-Galerkin (DPG) methods are new discontinuous Galerkin methods $[3,4,5,6,7,8]$ with interesting properties. In this article we consider a domain decomposition preconditioner for a DPG method for the Poisson problem.

Let $\Omega$ be a polyhedral domain in $\mathbb{R}^{d}(d=2,3), \Omega_{h}$ be a simplicial triangulation of $\Omega$. Following the notation in [8], the model Poisson problem (in an ultraweak formulation) is to find $\mathscr{U} \in U$ such that

$$
b(\mathscr{U}, \mathscr{V})=l(\mathscr{V}) \quad \forall \mathscr{V} \in V,
$$

where $U=\left[L_{2}(\Omega)\right]^{d} \times L_{2}(\Omega) \times H_{0}^{\frac{1}{2}}\left(\partial \Omega_{h}\right) \times H^{-\frac{1}{2}}\left(\partial \Omega_{h}\right), V=H\left(\operatorname{div} ; \Omega_{h}\right) \times H^{1}\left(\Omega_{h}\right)$,

$$
\begin{array}{r}
b(\mathscr{U}, \mathscr{V})=\int_{\Omega} \sigma \cdot \tau d x-\sum_{K \in \Omega_{h}} \int_{K} u \operatorname{div} \tau d x+\sum_{K \in \Omega_{h}} \int_{\partial K} \hat{u} \tau \cdot n d s \\
-\sum_{K \in \Omega_{h}} \int_{K} \sigma \cdot \operatorname{grad} v d x+\sum_{K \in \Omega_{h}} \int_{\partial K} v \hat{\sigma}_{n} d s
\end{array}
$$

for $\mathscr{U}=\left(\sigma, u, \hat{u}, \hat{\sigma}_{n}\right) \in U$ and $\mathscr{V}=(\tau, v) \in V$, and $l(\mathscr{V})=\int_{\Omega} f v d x$.

Here $H_{0}^{1 / 2}\left(\partial \Omega_{h}\right)$ (resp. $\left.H^{-1 / 2}\left(\partial \Omega_{h}\right)\right)$ is the subspace of $\prod_{K \in \Omega_{h}} H^{1 / 2}(\partial K)$ (resp. $\prod_{K \in \Omega_{h}} H^{-1 / 2}(\partial K)$ ) consisting of the traces of functions in $H_{0}^{1}(\Omega)$ (resp. traces of the normal components of vector fields in $H(\operatorname{div} ; \Omega)$ ), and $H\left(\operatorname{div} ; \Omega_{h}\right)$ (resp. $H^{1}\left(\Omega_{h}\right)$ ) is the space of piecewise $H(\mathrm{div})$ vector fields (resp. $H^{1}$ functions). The

${ }^{1}$ Department of Mathematics and Center for Computation and Technology, Louisiana State University, Baton Rouge, LA 70803, USA. andrewbemath.1su.edu, brenner@math.lsu.edu, sung@math.lsu.edu . ${ }^{2}$ Division of Computational Sciences in Mathematics, National Institute for Mathematical Sciences, Daejeon 305-811, South Korea. eunheepark@nims.re.kr 
inner product on $V$ is given by

$$
\left(\left(\tau_{1}, v_{1}\right),\left(\tau_{2}, v_{2}\right)\right)_{V}=\sum_{K \in \Omega_{h}} \int_{K}\left[\tau_{1} \cdot \tau_{2}+\operatorname{div} \tau_{1} \operatorname{div} \tau_{2}+v_{1} v_{2}+\operatorname{grad} v_{1} \cdot \operatorname{grad} v_{2}\right] d x
$$

The DPG method for the Poisson problem computes $\mathscr{U}_{h} \in U_{h}$ such that

$$
b\left(\mathscr{U}_{h}, \mathscr{V}\right)=l(\mathscr{V}) \quad \forall \mathscr{V} \in V_{h}
$$

Here the trial space $U_{h}(\subset U)$ is defined by

$$
U_{h}=\prod_{K \in \Omega_{h}}\left[P_{m}(K)\right]^{d} \times \prod_{K \in \Omega_{h}} P_{m}(K) \times \tilde{P}_{m+1}\left(\partial \Omega_{h}\right) \times P_{m}\left(\partial \Omega_{h}\right)
$$

$P_{m}(K)$ is the space of polynomials of total degree $\leq m$ on an element $K, \tilde{P}_{m+1}\left(\partial \Omega_{h}\right)=$ $H_{0}^{1 / 2}\left(\partial \Omega_{h}\right) \cap \prod_{K \in \Omega_{h}} \tilde{P}_{m+1}(\partial K)$, where $\tilde{P}_{m+1}(\partial K)$ is the restriction of $P_{m+1}(K)$ to $\partial K$, and $P_{m}\left(\partial \Omega_{h}\right)=H^{-1 / 2}\left(\partial \Omega_{h}\right) \cap \prod_{K \in \Omega_{h}} P_{m}(\partial K)$, where $P_{m}(\partial K)$ is the space of piecewise polynomials on the faces of $K$ with total degree $\leq m$.

Let $V^{r}=\left\{(\tau, v) \in V:\left.\tau\right|_{K} \in\left[P_{m+2}(K)\right]^{d},\left.v\right|_{K} \in P_{r}(K) \forall K \in \Omega_{h}\right\}$ for some $r \geq$ $m+d$. The discrete trial-to-test map $T_{h}: U_{h} \longrightarrow V^{r}$ is defined by

$$
\left(T_{h} \mathscr{U}_{h}, \mathscr{V}\right)_{V}=b\left(\mathscr{U}_{h}, \mathscr{V}\right), \quad \forall \mathscr{U}_{h} \in U_{h}, \mathscr{V} \in V^{r},
$$

and the test space $V_{h}$ is $T_{h} U_{h}$.

We can rewrite $(1)$ as $a_{h}\left(\mathscr{U}_{h}, \mathscr{W}\right)=l\left(T_{h} \mathscr{W}\right)$ for all $\mathscr{W} \in U_{h}$, where

$$
a_{h}(\mathscr{U}, \mathscr{W})=b_{h}\left(\mathscr{U}, T_{h} \mathscr{W}\right)=\left(T_{h} \mathscr{U}, T_{h} \mathscr{W}\right)_{V}
$$

is an SPD bilinear form on $V_{h} \times V_{h}$, and we define an operator $A_{h}: U_{h} \longrightarrow U_{h}^{\prime}$ by

$$
\left\langle A_{h} \mathscr{U}, \mathscr{W}\right\rangle=a_{h}(\mathscr{U}, \mathscr{W}) \quad \forall \mathscr{U}, \mathscr{W} \in U_{h}
$$

Our goal is to develop a one-level additive Schwarz preconditioner for $A_{h}$ (cf. [9]).

To avoid the proliferation of constants, we will use the notation $A \lesssim B$ (or $B \gtrsim A$ ) to represent the inequality $A \leq$ (constant) $\times B$, where the positive constant only depends on the shape regularity of $\Omega_{h}$ and the polynomial degrees $m$ and $r$. The notation $A \approx B$ is equivalent to $A \lesssim B$ and $B \lesssim A$.

A fundamental result in [8] is the equivalence

$$
a_{h}(\mathscr{U}, \mathscr{U}) \approx\|\sigma\|_{L_{2}(\Omega)}^{2}+\|u\|_{L_{2}(\Omega)}^{2}+\|\hat{u}\|_{H^{1 / 2}\left(\partial \Omega_{h}\right)}^{2}+\left\|\hat{\sigma}_{n}\right\|_{H^{-1 / 2}\left(\partial \Omega_{h}\right)}^{2}
$$

that holds for all $\mathscr{U}=\left(\sigma, u, \hat{u}, \hat{\sigma}_{n}\right) \in U_{h}$, where

$$
\begin{aligned}
\|\hat{u}\|_{H^{1 / 2}\left(\partial \Omega_{h}\right)}^{2} & =\sum_{K \in \Omega_{h}}\|\hat{u}\|_{H^{1 / 2}(\partial K)}^{2}=\sum_{K \in \Omega_{h}} \inf _{w \in H^{1}(K),\left.w\right|_{\partial K}=\hat{u}}\|w\|_{H^{1}(K)}^{2}, \\
\left\|\hat{\sigma}_{n}\right\|_{H^{-1 / 2}\left(\partial \Omega_{h}\right)}^{2} & =\sum_{K \in \Omega_{h}}\left\|\hat{\sigma}_{n}\right\|_{H^{-1 / 2}(\partial K)}^{2}=\sum_{K \in \Omega_{h}} \inf _{q \in H(\operatorname{div} ; K),\left.q \cdot n\right|_{\partial K}=\hat{\sigma}_{n}}\|q\|_{H(\operatorname{div} ; K)}^{2} .
\end{aligned}
$$


Therefore the analysis of domain decomposition preconditioners for $A_{h}$ requires a better understanding of the norms $\|\cdot\|_{H^{1 / 2}(\partial K)}$ and $\|\cdot\|_{H^{-1 / 2}(\partial K)}$ on the discrete spaces $\tilde{P}_{m+1}(\partial K)$ and $P_{m}(\partial K)$.

\section{Explicit Expressions for the Norms on $\tilde{P}_{m+1}(\partial K)$ and $P_{m}(\partial K)$}

Lemma 1. We have

$$
\|\tilde{\zeta}\|_{H^{1 / 2}(\partial K)}^{2} \approx h_{K}\left(\|\tilde{\zeta}\|_{L_{2}(\partial K)}^{2}+\sum_{F \in \Sigma_{K}}|\tilde{\zeta}|_{H^{1}(F)}^{2}\right) \quad \forall \tilde{\zeta} \in \tilde{P}_{m+1}(\partial K),
$$

where $h_{K}$ is the diameter of $K$ and $\Sigma_{K}$ is the set of the faces of $K$.

Proof. Let $\mathscr{N}(K)$ be the set of nodal points of the $P_{m}$ Lagrange finite element associated with $K$ and $\mathscr{N}(\partial K)$ be the set of points in $\mathscr{N}(K)$ that are on $\partial K$.

Given any $\tilde{\zeta} \in \tilde{P}_{m+1}(\partial K)$, we define $\tilde{\zeta}_{*} \in P_{m+1}(K)$ by

$$
\tilde{\zeta}_{*}(p)= \begin{cases}\tilde{\zeta}(p) & \text { if } p \in \mathscr{N}(\partial K), \\ \tilde{\zeta}_{\partial K} & \text { if } p \in \mathscr{N}(K) \backslash \mathscr{N}(\partial K),\end{cases}
$$

where $\tilde{\zeta}_{\partial K}$ is the mean value of $\tilde{\zeta}$ over $\partial K$. Since $\tilde{\zeta}_{*}=\tilde{\zeta}$ on $\partial K$, we have

$$
\|\tilde{\zeta}\|_{H^{1 / 2}(\partial K)}=\inf _{w \in H^{1}(K),\left.w\right|_{\partial K}=\zeta}\|w\|_{H^{1}(K)} \leq\left\|\tilde{\zeta}_{*}\right\|_{H^{1}(K)} .
$$

Suppose $w \in H^{1}(K)$ satisfies $w=\tilde{\zeta}$ on $\partial K$. It follows from (6) and the trace theorem with scaling that

$$
\left\|\tilde{\zeta}_{*}\right\|_{L_{2}(K)}^{2} \lesssim h_{K}\|\zeta\|_{L_{2}(\partial K)}^{2}=h_{K}\|w\|_{L_{2}(\partial K)}^{2} \lesssim\|w\|_{H^{1}(K)}^{2},
$$

and, by standard estimates,

$$
\begin{aligned}
\left|\tilde{\zeta}_{*}\right|_{H^{1}(K)}^{2}=\left|\tilde{\zeta}_{*}-\tilde{\zeta}_{\partial K}\right|_{H^{1}(K)}^{2} & \lesssim h_{K}^{-1}\left\|\tilde{\zeta}_{*}-\tilde{\zeta}_{\partial K}\right\|_{L_{2}(\partial K)}^{2} \\
& =h_{K}^{-1}\left\|w-w_{\partial K}\right\|_{L_{2}(\partial K)}^{2} \lesssim|w|_{H^{1}(K)}^{2} .
\end{aligned}
$$

Combining (7)-(9), we have $\|\tilde{\zeta}\|_{H^{1 / 2}(\partial K)}^{2} \approx\left\|\tilde{\zeta}_{*}\right\|_{H^{1}(K)}^{2}$. The lemma then follows from (6), the equivalence of norms on finite dimensional spaces and scaling.

Lemma 2. We have

$$
\|\zeta\|_{H^{-1 / 2}(\partial K)}^{2} \approx h_{K}\|\zeta\|_{L_{2}(\partial K)}^{2}+h_{K}^{-d}\left(\int_{\partial K} \zeta d s\right)^{2} \quad \forall \zeta \in P_{m}(\partial K) .
$$


Proof. We begin with the reference simplex $\hat{K}$. Let $R T_{m}(\hat{K})$ be the $m$-th order Raviart-Thomas space (cf. [2]). Given any $\zeta \in P_{m}(\partial \hat{K})$, we introduce a (nonempty) subspace $R T_{m}(\hat{K}, \zeta)=\left\{q \in R T_{m}(\hat{K}): q \cdot n=\zeta\right.$ on $\partial \hat{K}$ and $\left.\operatorname{div} q \in P_{0}(\hat{K})\right\}$ of $R T_{m}(\hat{K})$.

Let $\zeta_{*} \in R T_{m}(\hat{K}, \zeta)$ be defined by

$$
\zeta_{*}=\min _{q \in R T_{m}(\hat{K}, \zeta)}\|q\|_{L_{2}(\hat{K})} .
$$

Then the map $\hat{S}: P_{m}(\partial \hat{K}) \longrightarrow R T_{m}(\hat{K})$ that maps $\zeta$ to $\zeta_{*}$ is linear and one-to-one, and we have $(\hat{S} \zeta) \cdot n=\zeta$ on $\partial \hat{K}, \operatorname{div}(\hat{S} \zeta) \in P_{0}(\hat{K})$ and

$$
\|\hat{S} \zeta\|_{L_{2}(\hat{K})} \approx\|\zeta\|_{L_{2}(\partial \hat{K})} \quad \forall \zeta \in P_{m}(\partial \hat{K}) .
$$

Let $\zeta_{1}, \ldots, \zeta_{N_{m}}$ be a basis of $P_{m}(\partial \hat{K})$ and $1=\phi_{1}, \ldots, \phi_{N_{m}} \in H^{1 / 2}(\partial \hat{K})$ satisfy $\operatorname{det}\left[\int_{\partial \hat{K}} \zeta_{i} \phi_{j} d \hat{s}\right]_{1 \leq i, j \leq N_{m}} \neq 0$. We define the map $\hat{Q}: H(\operatorname{div} ; \hat{K}) \longrightarrow P_{m}(\partial \hat{K})$ by

$$
\int_{\partial \hat{K}}(\hat{Q} q) \phi_{j} d \hat{s}=\left\langle q \cdot n, \phi_{j}\right\rangle_{H^{-1 / 2}(\partial \hat{K}) \times H^{1 / 2}(\partial \hat{K})} \quad \text { for } \quad 1 \leq j \leq N_{m} .
$$

It follows from the definition of $\hat{Q}$ that $\left\|\hat{Q}_{q}\right\|_{L_{2}(\partial \hat{K})} \lesssim\|q\|_{H(\mathrm{div} ; \hat{K})}$ for all $q \in$ $H(\operatorname{div} ; \hat{K})$, and $\hat{Q} q=\zeta$ if $q \cdot n=\zeta \in P_{m}(\partial \hat{K})$, in which case

$$
\|\hat{S} \zeta\|_{L_{2}(\hat{K})} \lesssim\|\zeta\|_{L_{2}(\partial \hat{K})}=\|\hat{Q} q\|_{L_{2}(\partial \hat{K})} \lesssim\|q\|_{H(\operatorname{div} ; \hat{K})} .
$$

Moreover, since $\phi_{1}=1$, we have

$$
\int_{\hat{K}} \operatorname{div}(\hat{S} \zeta) d \hat{x}=\int_{\partial \hat{K}}(\hat{Q} q) 1 d \hat{s}=\langle q \cdot n, 1\rangle_{H^{-1 / 2}(\partial \hat{K}) \times H^{1 / 2}(\partial \hat{K})}=\int_{\hat{K}} \operatorname{div} q d \hat{x}
$$

and hence

$$
\|\operatorname{div}(\hat{S} \zeta)\|_{L_{2}(\hat{K})} \lesssim\|\operatorname{div} q\|_{L_{2}(\hat{K})} .
$$

Now we turn to a general simplex $K$. It follows from (10)-(12) and standard properties of the Piola transform for $H$ (div) (cf. [10]) that there exists a linear map $S: P_{m}(\partial K) \longrightarrow R T_{m}(K)$ with the following properties:

(i) $(S \zeta) \cdot n=\zeta$ and hence

$$
\|\zeta\|_{H^{-1 / 2}(\partial K)}=\inf _{q \in H(\operatorname{div} ; K),\left.q \cdot n\right|_{\partial K}=\zeta}\|q\|_{H(\mathrm{div} ; K)} \leq\|S \zeta\|_{H(\mathrm{div} ; K)} \quad \forall \zeta \in P_{m}(\partial K),
$$

(ii) for any $q \in H(\operatorname{div} ; K)$ such that $q \cdot n=\zeta$, we have

$$
\|S \zeta\|_{H(\operatorname{div} ; K)} \lesssim\|q\|_{H(\mathrm{div} ; K)},
$$

(iii) $\operatorname{div}(S \zeta) \in P_{0}(K)$ and hence

$$
\int_{K} \operatorname{div}(S \zeta) d x=\int_{\partial K} \zeta d s \quad \text { or } \quad\|\operatorname{div}(S \zeta)\|_{L_{2}(K)}^{2}=\left(\int_{\partial K} \zeta d s\right)^{2} /|K|,
$$


(iv) we have

$$
h_{K}^{-d}\|S \zeta\|_{L_{2}(K)}^{2} \approx h_{K}^{-(d-1)}\|\zeta\|_{L_{2}(\partial K)}^{2}
$$

Properties (i)-(iv) then imply

$$
\|\zeta\|_{H^{-1 / 2}(\partial K)}^{2} \approx\|S \zeta\|_{H(\operatorname{div} ; K)}^{2} \approx h_{K}\|\zeta\|_{L_{2}(\partial K)}^{2}+h_{K}^{-d}\left(\int_{\partial K} \zeta d s\right)^{2} .
$$

\section{A Domain Decomposition Preconditioner}

Let $\Omega$ be partitioned into overlapping subdomains $\Omega_{1}, \ldots, \Omega_{J}$ that are aligned with $\Omega_{h}$. The overlap among the subdomains is measured by $\delta$ and we assume (cf. [11]) there is a partition of unity $\theta_{1}, \ldots, \theta_{J} \in C^{\infty}(\bar{\Omega})$ that satisfies the usual properties: $\theta_{j} \geq 0, \sum_{j=1}^{J} \theta_{j}=1$ on $\bar{\Omega}, \theta_{j}=0$ on $\Omega \backslash \Omega_{j}$, and

$$
\left\|\nabla \theta_{j}\right\|_{L_{\infty}(\Omega)} \lesssim \delta^{-1} \quad \forall 1 \leq j \leq J
$$

We take the subdomain space to be $U_{j}=\left\{\mathscr{U} \in U_{h}: \mathscr{U}=0\right.$ on $\left.\Omega \backslash \Omega_{j}\right\}$. Let $\mathscr{U}=$ $\left(\sigma, u, \hat{u}, \hat{\sigma}_{n}\right) \in U_{h}$. Then $\mathscr{U} \in U_{j}$ if and only if (i) $\sigma$ and $u$ vanish on every $K$ outside $\Omega_{j}$ and (ii) $\hat{u}$ and $\hat{\sigma}_{n}$ vanish on $\partial K$ for every $K$ outside $\Omega_{j}$. We define $a_{j}(\cdot, \cdot)$ to be the restriction of $a_{h}(\cdot, \cdot)$ on $U_{j} \times U_{j}$. Let $A_{j}: U_{j} \longrightarrow U_{j}^{\prime}$ be defined by

$$
\left\langle A_{j} \mathscr{U}_{j}, \mathscr{W}_{j}\right\rangle=a_{j}\left(\mathscr{U}_{j}, \mathscr{W}_{j}\right) \quad \forall \mathscr{U}_{j}, \mathscr{W}_{j} \in U_{j} .
$$

It follows from (3) that

$$
a_{j}\left(\mathscr{R}_{j}, \mathscr{U}_{j}\right) \approx\left\|\sigma_{j}\right\|_{L_{2}\left(\Omega_{j}\right)}^{2}+\left\|u_{j}\right\|_{L_{2}\left(\Omega_{j}\right)}^{2}+\left\|\hat{u}_{j}\right\|_{H^{1 / 2}\left(\partial \Omega_{j, h}\right)}^{2}+\left\|\hat{\sigma}_{n, j}\right\|_{H^{-1 / 2}\left(\partial \Omega_{j, h}\right)}^{2},
$$

where $\mathscr{U}_{j}=\left(\sigma_{j}, u_{j}, \hat{u}_{j}, \hat{\sigma}_{n, j}\right) \in U_{j}, \Omega_{j, h}$ is the triangulation of $\Omega_{j}$ induced by $\Omega_{h}$ and the norms $\|\cdot\|_{H^{1 / 2}\left(\partial \Omega_{j, h}\right)}$ and $\|\cdot\|_{H^{-1 / 2}\left(\partial \Omega_{j, h}\right)}$ are analogous to those in (4) and (5).

Let $I_{j}: U_{j} \longrightarrow U_{h}$ be the natural injection. The one-level additive Schwarz preconditioner $B_{h}: U_{h}^{\prime} \longrightarrow U_{h}$ is defined by

$$
B_{h}=\sum_{j=1}^{J} I_{j} A_{j}^{-1} I_{j}^{t}
$$

Lemma 3. We have

$$
\lambda_{\min }\left(B_{h} A_{h}\right) \gtrsim \delta^{2}
$$

Proof. Let $I_{h, 1}, I_{h, 2}, I_{h, 3}$ and $I_{h, 4}$ be the nodal interpolation operators for the components $\prod_{K \in \Omega_{h}}\left[P_{m}(K)\right]^{d}, \prod_{K \in \Omega_{h}} P_{m}(K), \tilde{P}_{m+1}\left(\partial \Omega_{h}\right)$ and $P_{m}\left(\partial \Omega_{h}\right)$ of $U_{h}$ respectively. Given any $\mathscr{U}=\left(\sigma, u, \hat{u}, \hat{\sigma}_{n}\right) \in U_{h}$, we define $\mathscr{\mathscr { N }} \in U_{j}$ by

$$
\mathscr{U}_{j}=\left(I_{h, 1}\left(\theta_{j} \sigma\right), I_{h, 2}\left(\theta_{j} u\right), I_{h, 3}\left(\theta_{j} \hat{u}\right), I_{h, 4}\left(\theta_{j} \hat{\sigma}_{n}\right)\right) .
$$


Then we have $\mathscr{U}=\sum_{j=1}^{J} \mathscr{\mathscr { H }} j$ and, in view of (14) and (15),

$$
\begin{aligned}
\left\langle A_{j} \mathscr{U l}_{j}, \mathscr{I}_{j}\right\rangle \approx\left\|I_{h, 1}\left(\theta_{j} \sigma\right)\right\|_{L_{2}\left(\Omega_{j}\right)}^{2}+\left\|I_{h, 2}\left(\theta_{j} u\right)\right\|_{L_{2}\left(\Omega_{j}\right)}^{2} & \\
& +\left\|I_{h, 3}\left(\theta_{j} \hat{u}\right)\right\|_{H^{1 / 2}\left(\partial \Omega_{j, h}\right)}^{2}+\left\|I_{h, 4}\left(\theta_{j} \hat{\sigma}_{h}\right)\right\|_{H^{-1 / 2}\left(\partial \Omega_{j, h}\right.}^{2} .
\end{aligned}
$$

The following bounds for the first two terms on the right-hand side of (16) are straightforward:

$$
\left\|I_{h, 1}\left(\theta_{j} \sigma\right)\right\|_{L_{2}\left(\Omega_{j}\right)}^{2} \lesssim\|\sigma\|_{L_{2}\left(\Omega_{j}\right)}^{2} \quad \text { and } \quad\left\|I_{h, 2}\left(\theta_{j} u\right)\right\|_{L_{2}\left(\Omega_{j}\right)}^{2} \lesssim\|u\|_{L_{2}\left(\Omega_{j}\right)}^{2} .
$$

We will use Lemma 1 and Lemma 2 to derive the following bounds

$$
\begin{aligned}
\left\|I_{h, 3}\left(\theta_{j} \hat{u}\right)\right\|_{H^{1 / 2}\left(\partial \Omega_{j, h}\right)}^{2} & \lesssim \delta^{-2}\|\hat{u}\|_{H^{1 / 2}\left(\partial \Omega_{j, h}\right)}^{2}, \\
\left\|I_{h, 4}\left(\theta_{j} \hat{\sigma}_{n}\right)\right\|_{H^{-1 / 2}\left(\partial \Omega_{j, h}\right)}^{2} & \lesssim \delta^{-2}\left\|\hat{\sigma}_{n}\right\|_{H^{-1 / 2}\left(\partial \Omega_{j, h}\right)}^{2} .
\end{aligned}
$$

Let $K \in \Omega_{j, h}$. It follows from Lemma 1, (13) and standard discrete estimates that

$$
\begin{aligned}
\left\|I_{h, 3}\left(\theta_{j} \hat{u}\right)\right\|_{H^{1 / 2}(\partial K)}^{2} \approx h_{K}\left(\left\|I_{h, 3}\left(\theta_{j} \hat{u}\right)\right\|_{L_{2}(\partial K)}^{2}+\sum_{F \in \Sigma_{K}}\left|I_{h, 3}\left(\theta_{j} \hat{u}\right)\right|_{H^{1}(F)}^{2}\right) \\
\quad \lesssim h_{K}\|\hat{u}\|_{L_{2}(\partial K)}^{2}+h_{K} \sum_{F \in \Sigma_{K}}\left(\left\|\nabla \theta_{j}\right\|_{L_{\infty}(\Omega)}^{2}\|\hat{u}\|_{L_{2}(F)}^{2}+\left\|\theta_{j}\right\|_{L_{\infty}(\Omega)}^{2}|\hat{u}|_{H^{1}(F)}^{2}\right) \\
\quad \lesssim h_{K}\|\hat{u}\|_{L_{2}(\partial K)}^{2}+h_{K} \delta^{-2}\|\hat{u}\|_{L_{2}(\partial K)}^{2}+h_{K} \sum_{F \in \Sigma_{K}}|\hat{u}|_{H^{1}(F)}^{2} \lesssim \delta^{-2}\|\hat{u}\|_{H^{1 / 2}(\partial K)}^{2} .
\end{aligned}
$$

Summing up this estimate over all the simplexes in $\Omega_{j, h}$ yields (18).

Similarly, it follows from Lemma 2 and (13) that

$$
\begin{aligned}
& \left\|I_{h, 4}\left(\theta_{j} \hat{\sigma}_{n}\right)\right\|_{H^{-1 / 2}(\partial \hat{K})}^{2} \approx h_{K}\left\|I_{h, 4}\left(\theta_{j} \hat{\sigma}_{n}\right)\right\|_{L_{2}(\partial K)}^{2}+h_{K}^{-d}\left(\int_{\partial K} I_{h, 4}\left(\theta_{j} \hat{\sigma}_{n}\right) d s\right)^{2} \\
& \quad \lesssim h_{K}\left\|\hat{\sigma}_{n}\right\|_{L_{2}(\partial K)}^{2}+h_{K}^{-d}\left(\int_{\partial K} I_{h, 4}\left[\left(\theta_{j}-\theta_{j}^{K}\right) \hat{\sigma}_{n}\right] d s\right)^{2}+h_{K}^{-d} \theta_{j}^{K}\left(\int_{\partial K} \hat{\sigma}_{n} d s\right)^{2} \\
& \quad \lesssim h_{K}\left\|\hat{\sigma}_{n}\right\|_{L_{2}(\partial K)}^{2}+h_{K} \delta^{-2}\left\|\hat{\sigma}_{n}\right\|_{L_{2}(\partial K)}^{2}+h_{K}^{-d}\left(\int_{\partial K} \hat{\sigma}_{n} d s\right)^{2} \lesssim \delta^{-2}\left\|\hat{\sigma}_{n}\right\|_{H^{-1 / 2}(\partial K)}^{2},
\end{aligned}
$$

where $\theta_{j}^{K}$ is the mean value of $\sigma_{j}$ over $K$. Summing up this estimate over all the simplexes in $\Omega_{j, h}$ gives us (19).

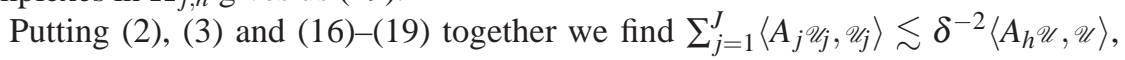
which implies $\lambda_{\min }\left(B_{h} A_{h}\right) \gtrsim \delta^{2}$ by the standard theory of additive Schwarz preconditioners [11].

Combining Lemma 3 with the standard estimate $\lambda_{\max }\left(B_{h} A_{h}\right) \lesssim 1$, we obtain the following theorem. 
Theorem 1. We have

$$
\kappa\left(B_{h} A_{h}\right)=\frac{\lambda_{\max }\left(B_{h} A_{h}\right)}{\lambda_{\min }\left(B_{h} A_{h}\right)} \leq C \delta^{-2},
$$

where the positive constant $C$ depends only on the shape regularity of $\Omega_{h}$ and the polynomial degrees $m$ and $r$.

Remark 1. Theorem 1 is also valid for DPG methods based on tensor product finite elements.

\section{Numerical results}

We solve the Poisson problem on the square $(0,1)^{2}$ with exact solution $u=$ $\sin \left(\pi x_{1}\right) \sin \left(\pi x_{2}\right)$ and uniform square meshes. The trial space is based on $Q_{1}$ polynomials for $\sigma$ and $u, P_{2}$ polynomials for $\hat{u}$, and $P_{1}$ polynomials for $\hat{\sigma}_{n}$. We use bicubic polynomials for the space $V^{r}$ in the construction of the trial-to-test map $T_{h}$.

The number of conjugate gradient iterations required to reduce the residual by $10^{10}$ are given in Table 1 for four overlapping subdomains. The linear growth of the number of iterations for the unpreconditioned system is consistent with the condition number estimate $\kappa\left(A_{h}\right) \lesssim h^{-2}$ in [8]. Note that in this case the boundary of every subdomain has a nonempty intersection with $\partial \Omega$ and it is not difficult to use a discrete Poincaré inequality to show that the estimate in Theorem 1 can be improved to $\kappa\left(B_{h} A_{h}\right) \lesssim|\ln h| \delta^{-1}$. This is consistent with the observed growth of the number of iterations for the preconditioned system as $\delta$ decreases.

Table 1 Number of iterations for the Schwarz preconditioner with subdomain size $H=1 / 2$.

\begin{tabular}{ll|ll}
\hline \multicolumn{1}{l}{$h$} & \multicolumn{1}{l|}{$\delta$} & unpreconditioned preconditioned \\
\hline $2^{-2}$ & $2^{-2}$ & 496 & 14 \\
$2^{-3}$ & $2^{-3}$ & 1556 & 17 \\
& $2^{-2}$ & & 14 \\
$2^{-4}$ & $2^{-4}$ & 3865 & 20 \\
& $2^{-3}$ & & 17 \\
& $2^{-2}$ & & 14 \\
$2^{-5}$ & $2^{-5}$ & 8793 & 27 \\
& $2^{-4}$ & & 20 \\
& $2^{-3}$ & 18 \\
\hline
\end{tabular}

In Table 2 we display the results for $h=2^{-5}$ and various subdomain sizes $H$ with $\delta=H / 2$. The estimate $\kappa\left(B_{h} A_{h}\right) \lesssim \delta^{-2} \approx H^{-2}$ is consistent with the observed linear growth of the number of iterations for the preconditioned system as $H$ decreases. Such a condition number estimate for the one-level additive Schwarz preconditioner is known to be sharp for standard finite element methods [1]. 
Table 2 Number of iterations with $h=2^{-5}$ and various subdomain sizes $H$ with $\delta=H / 2$.

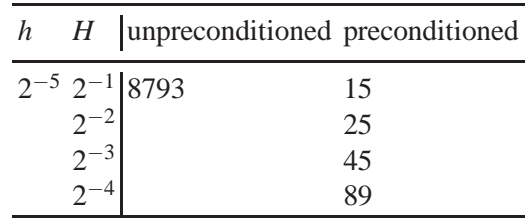

Acknowledgements The work of the first author was supported in part by the National Science Foundation VIGRE Grant DMS-07-39382. The work of the second and fourth authors was supported in part by the National Science Foundation under Grant No. DMS-10-16332. The work of the third author was supported in part by a KRCF research fellowship for young scientists. The authors would also like to thank Leszek Demkowicz for helpful discussions.

\section{References}

[1] Brenner, S.C.: Lower bounds in domain decomposition. In: Domain Decomposition Methods in Science and Engineering XVI, pp. 27-39. Springer, Berlin (2007)

[2] Brezzi, F., Fortin, M.: Mixed and Hybrid Finite Element Methods. SpringerVerlag, New York-Berlin-Heidelberg (1991)

[3] Demkowicz, L., Gopalakrishnan, J.: A class of discontinuous Petrov-Galerkin methods. Part I: The transport equation. Comp. Meth. Appl. Math. Engrg. 199, 1558-1572 (2010)

[4] Demkowicz, L., Gopalakrishnan, J.: Analysis of the DPG method for the Poisson equation. SIAM J. Numer. Anal. 49, 1788-1809 (2011)

[5] Demkowicz, L., Gopalakrishnan, J.: A class of discontinuous Petrov-Galerkin methods. Part II: Optimal test functions. Num. Meth. Part. Diff. Eq. 27, 70105 (2011)

[6] Demkowicz, L., Gopalakrishnan, J.: A class of discontinuous Petrov-Galerkin methods. Part IV: The optimal test norm and time-harmonic wave propagation in 1D. J. Comp. Phys. 230, 2406-2432 (2011)

[7] Demkowicz, L., Gopalakrishnan, J., Niemi, A.H.: A class of discontinuous Petrov-Galerkin methods. Part III: Adaptivity. Appl. Numer. Math. (to appear)

[8] Gopalakrishnan, J., Qiu, W.: An analysis of the practical DPG method. Math. Comp. (to appear)

[9] Matsokin, A., Nepomnyaschikh, S.: A Schwarz alternating method in a subspace. Soviet Math. 29, 78-84 (1985)

[10] Monk, P.: Finite Element Methods for Maxwell's Equations. Oxford University Press, New York (2003)

[11] Toselli, A., Widlund, O.: Domain Decomposition Methods - Algorithms and Theory. Springer, New York (2005) 